\title{
CFD Simulation of Particles Effects on Characteristics of Detonation
}

\author{
Hesam Shafiee and Mohammad Hassan Djavareshkian, Member, IACSIT
}

\begin{abstract}
This paper covers the topic of the interaction process between a detonation wave and a cloud of inert particles. A computer code based on numerical method on Flux Corrected Transport algorithm was developed to solve the Euler equations for inviscid flow. The chemical reaction is modeled with one-step Arrhenius chemistry, and the particle phase is modeled in Lagrangian frame of reference. Simulation results illustrate that detonation wave could be suppressed by a cloud of inert particle, if the cloud parameters meet some critical conditions.
\end{abstract}

Index Terms_-Detonation, modeling, particles, suppression.

\section{INTRODUCTION}

Accidental explosions constitute a problem in various industries involving combustion dust, liquid and gas [1]. To control accidental explosions, various systems are used for explosion mitigation and suppression. One such system is explosion suppression by addition of suspended chemically inert particles. In order to develop more effective suppression systems, the interaction process between a detonation wave and a cloud of particles needs to be investigated. For small dimensional situation, 2D simulation can be used to qualitatively investigate the detonation-particle interaction process. Bioko et al. [2] performed experimental and numerical investigations regarding shock wave interaction with a cloud of particles. Wang et al. [3] studied the numerical modeling of near-wall two moving at constant speed. Parmar et al. [4] proposed a model of the unsteady force shock-particle interaction. Oran et al. [5] performed a numerical study of a two-dimensional Hydrogen- Oxygen detonation using detailed chemistry. Gamezo et al. [6] carried a numerical study on two-dimensional reactive flow dynamics in cellular detonation waves. Carvel et al. [7] showed experimental studies on the effect of both inert and reactive particles on the pressure profile of a detonation.

The Lagrangian modeling of particle flow is not very common in detonation research. Still a few examples can be mentioned: Ruggirello et al. [8] used Lagrangian description of particles in their investigation of particle compressibility and ignition from shock focusing. Cheatham and Kailasanath [9] also used Lagrangian description of droplets in their investigation of liquid fuelled detonations.

Manuscript received November 4, 2013; revised March 3, 2014.

The authors are with the Mechanical Engineering Department of Ferdowsi University of Mashhad, Iran (e-mail: shafiee.hesam@gmail.com, javareshkian@um.ac.ir).

\section{MAthematicAl Model}

\section{A. Gas Phase Modeling}

For gas phase, the influence of viscosity is low then the Euler equations with the ideal gas law can be used and written as:

$$
\begin{gathered}
\frac{\partial \rho}{\partial t}+\nabla \cdot(\rho \bar{u})=0 \\
\frac{\partial(\rho \bar{u})}{\partial t}+\nabla \cdot((\rho \bar{u}) \otimes \bar{u})+\nabla P=0 \\
\frac{\partial E}{\partial t}+\nabla \cdot(E \bar{u})+\nabla P \bar{u}=0 \\
P=\frac{\rho R T}{M}
\end{gathered}
$$

where $u$ is the velocity, $\rho$ is density, $E$ is the total energy, $T$ is temperature, $R$ is the ideal gas constant, $M$ is the molar mass and $P$ is pressure. These equations can be modified by adding source terms to calculate particle interaction and chemical reactions. The gas temperature $T$ is linked to the energy of the gas by the polytropic equation of state, kinetic energy and chemical energy. (see Section $B$ ):

$$
\mathrm{E}=\frac{\rho R T}{M(\gamma-1)}+\frac{\rho u^{2}}{2}-\alpha \rho Q_{r}
$$

where $\gamma$ is the polytropic exponent and $Q_{r}$ is the heat of chemical reaction per unit mass.

We neglect turbulence modeling because in the propagation of a detonation wave, the stage of the turbulent flame development and propagation is of minor importance compared to the fast energy deposition in the spontaneous ignition front [10].

\section{B. Chemical Reaction}

Reaction model that we use here is adapted from Gamezo et al. [6].The reaction process variable $\alpha$ is used to model a simple one step reaction, which will go from zero(only reactions) to one(only products). Time rate of change of $\alpha$ will be governed by:

$$
\frac{\partial \rho \alpha}{\partial t}+\nabla \cdot(\alpha \rho \bar{u})-\rho W=0
$$

where $\alpha$ is the reaction coefficient and $W$ is the chemical rate that defined by the Arrhenius law:

$$
w=\frac{\partial \rho}{\partial t} A(1-\alpha) e^{-A r}=0
$$


where $A r$ is the Arrhenius number:

$$
A r=\frac{E_{a}}{R T}
$$

where $a$ is the pre- exponential reaction rate factor, $E_{a}$ is the activation energy.

\section{Particle-Gas Interaction}

In simulation of two phase flow, there are three main approaches, namely the Eulerian-Eulerian (E-E), the Eulerian- Lagrangian (E-L), and the direct numerical simulation (DNS) approach. Since the purpose is to investigate the effect of particles on a detonation wave the E-L approach has been used in this research. By considering low particle concentration (volume fraction $0.1 \%$ as shown later), inter- particle collisions and porosity can be neglect.

The particles in the system are treated in Lagrangian frame of reference. This means that each individual particle trajectory can be calculated by applying Newton's second law of motion to the particle:

$$
m_{p} \frac{d \vec{u}_{p}}{d t}=\sum \vec{F}_{i}
$$

where $\sum \vec{F}_{i}$ is the total force acting on the particle from a number of sources and $m_{p}=\frac{\rho_{p} d_{p} \pi}{6}$ is the mass of the particle where $\rho_{p}$ is particle mass density and $d_{p}$ is diameter).

The drag force acting on the particle is determined by the equation [11]:

$$
\vec{F}_{d}=C_{d} \rho A_{p} \frac{\left|u-u_{p}\right|\left(u-u_{p}\right)}{2}
$$

where $C_{d}$ is drag coefficient, $A_{p}$ is projected particle area and $u_{p}$ is particle velocity. The drag coefficient $C_{d}$ is calculated using an equation cited and validated by Bioko et al. [3]:

$$
C_{d}(\operatorname{Re}, M a)=\left(1+\exp \left(\frac{-0.43}{M a^{4.67}}\right)\right) \cdot\left(0.38+\frac{24}{\operatorname{Re}}+\frac{4}{\sqrt{\mathrm{Re}}}\right)
$$

where $\operatorname{Re}$ is particle Reynolds number:

$$
\operatorname{Re}=\frac{\rho d_{p}\left|u-u_{p}\right|}{\mu}
$$

with $\mu$ being the dynamic viscosity and $M a$ is Mach number:

$$
M a=\frac{\sqrt{\rho}\left|u-u_{p}\right|}{\sqrt{2 p}}
$$

The heat transfer rate from the gas to the particle is determined by the equation [11, p. 102]

$$
Q_{T}=N u \pi d_{p} k_{g}\left(T-T_{p}\right)
$$

where $N u$ is the Nusselt number, $k_{g}$ is the thermal conduction coefficient of gas and $T_{p}$ is particle temperature.

$$
N u=2+0.6 \sqrt{\operatorname{Re}} \operatorname{Pr}^{1 / 3}
$$

where $\operatorname{Pr}$ is prandtl number:

$$
P_{r}=\frac{\mu C_{p, g}}{k_{g}}
$$

In the above: $C_{p, g}$ is the specific heat capacity of gas at constant pressure. In the present studies, heat exchange caused by radiation effects was neglected.

\section{Dynamic Viscosity}

Some work has been done to develop a model for calculating viscosity in detonation products [12]. Balapanov et al. [13] mentions using the Chapman-Enskog theory to calculate the dynamic viscosity in their investigations. Papalexandris [14] uses a simplified version of the Sutherland formula [15, p. 233]. In this research, without loss of generality, the dynamic viscosity in the present calculations was held constant and equal to $\mu=10^{-5}$ (Pas).

\section{VALIDATION}

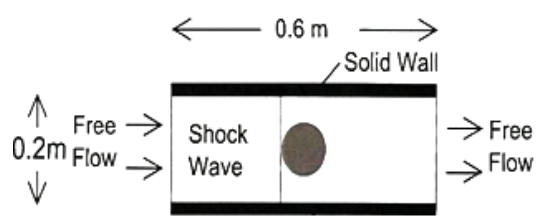

Fig. 1. The initial setup of the particle cloud shock wave interaction simulation.

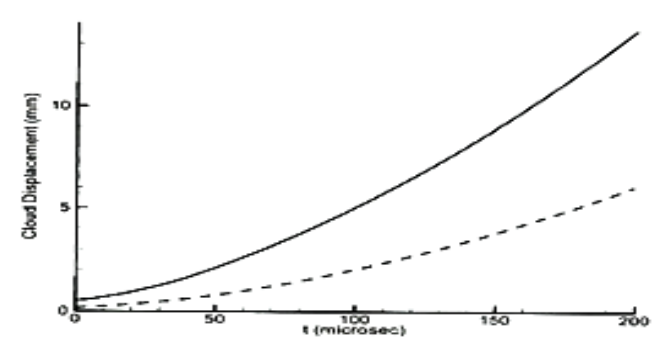

(a)

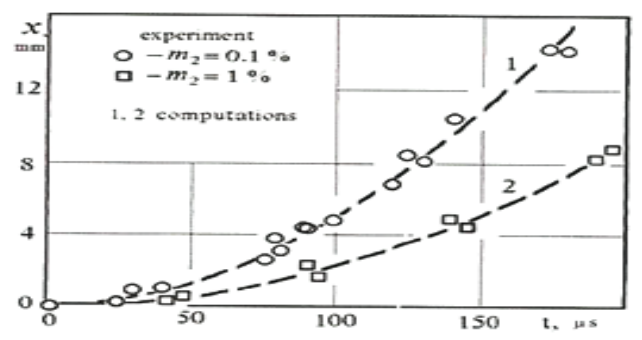

(b)

Fig. 2. Cloud displacement as a function of time for two particle volume fraction in the initial cloud. Solid line $=0.1 \%$, dashed line $=1 \%$ initial volume fraction in the cloud. The Mach-number of the shock wave was 2.8 (a). Cloud displacement from Bioko et al. The lines are computational values, and the dots are experimental data (b).

Validate the particle model, a simulation of a shock wave colliding with a circular, uniformly the distributed particle cloud was carried out. The initial conditions of the simulation were chosen in agreement with the experimental setup done by Bioko et al. [2] . The gas properties were set to those of air at room temperature and atmospheric pressure. The particle had mass density $\rho=8600\left(\mathrm{~kg} / \mathrm{m}^{3}\right)$ and diameter 
$d_{p}=130(\mu m)$ The initial shock wave was a square profile with pressure $p=15 p_{0}$ and $M a=2.8$. The initial mass density of the gas was constant over the entire domain. A schematic view of the initial setup can be found in Fig. 1. The cloud displacement was calculated as the main difference in position of each particle the $\mathrm{x}$-direction. Fig. 2 compares the cloud displacement as a function of time with corresponding from Bioko et al. There is very good agreement in the case of initial particle volume fraction $0.1 \%$.

\section{Simulation Results}

\section{A. Condition}

In all simulation with particles, a $500 \times 300$ grid was used (the grid independency was assured by running simulations of different grids). The initial conditions were the same as those shown in Table I. The detonation was initiated by using 50 cell wide region with high pressure and near the left boundary at $t_{0}$. The shock wave was

then propagated to the right boundary. Here, the grid velocity was adjusted, so that the grid velocity matched the shock velocity. As a part of the initial setup, random velocities in the order of $10^{-3}(\mathrm{~m} / \mathrm{s})$ were added to the $\mathrm{y}$-component of the gas velocity in each cell.

TABLE I: INITIAL PARAMETERS. * THE $u_{y}$ VALUES WERE RANDOM

\begin{tabular}{|c|c|c|c|c|c|}
\hline- & $P(\mathrm{~atm})$ & $\rho\left(\mathrm{kg} / \mathrm{m}^{2}\right)$ & $\alpha$ & $u_{x}(\mathrm{~m} / \mathrm{s})$ & $u_{y}(\mathrm{~m} / \mathrm{s})$ \\
\hline $\begin{array}{c}\text { High } \\
\text { Pressure }\end{array}$ & 40 & 0.49 & 1 & 0 & $10^{-3} *$ \\
\hline $\begin{array}{c}\text { Low } \\
\text { Pressure }\end{array}$ & 1 & 0.49 & 0 & 0 & $10^{-3} *$ \\
\hline
\end{tabular}

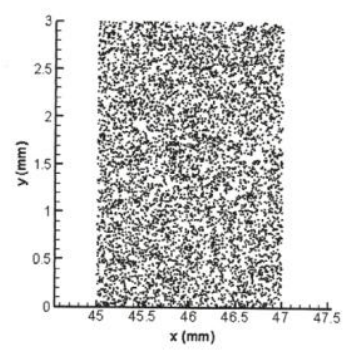

Fig. 3. Initial particle cloud for the standard setup.

In addition, a particle cloud was placed between $x=45-47$ mm (Fig. 3). The maximum pressure plot of the standard setup is found in Fig. 4 (a). A weak cellular pattern is observed, but this is most likely some kind of residual effect from the pattern in the original detonation. Fig. 4(b) shows the maximum pressure of the detonation averaged in the y-direction.

It is clear that the particles have a substantial mitigating effect on the detonation wave. A large drop in pressure happens inside the particle cloud, and the pressure is slightly decreasing after the cloud.

Fig. 5 shows a plot of the reaction coefficient and pressure after the wave has hit the particle cloud for three different points in time, with a time step of $2.5(\mu \mathrm{s})$ between them. The reaction front is slowing down, and a gap between the pressure front and the reaction front is established. In the third frame, unreached gas is exiting the computational domain at the left boundary. When this happens, the solution is invalidated because the entire reaction process is not calculated. It looks like the detonation is suppressed, since the pressure is gradually decreasing, but the possibility of re-ignition can not be excluded.

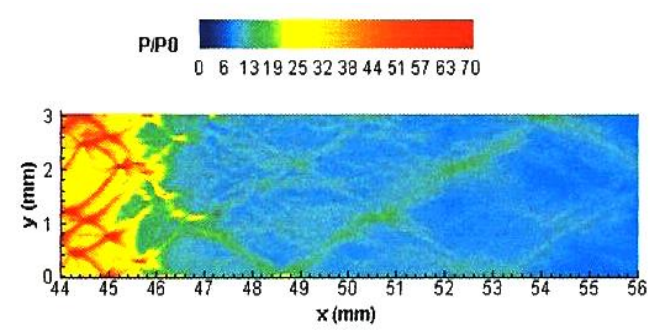

(a)

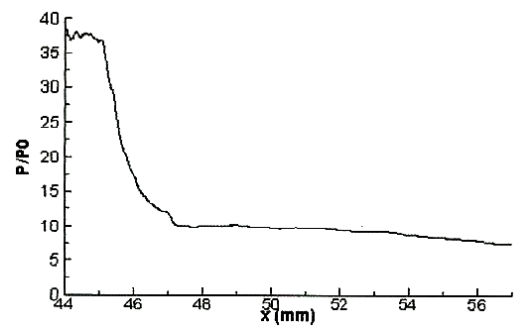

(b)

Fig. 4. Maximum pressure plot of the standard setup. The particle cloud is a belt of particles evenly distributed between $x=45 \mathrm{~mm}$ and $x=47 \mathrm{~mm}$, and initial particle volume fraction $0.1 \%$.(a). Maximum pressure for the standard setup, averaged in the $y$ - dimension (b).

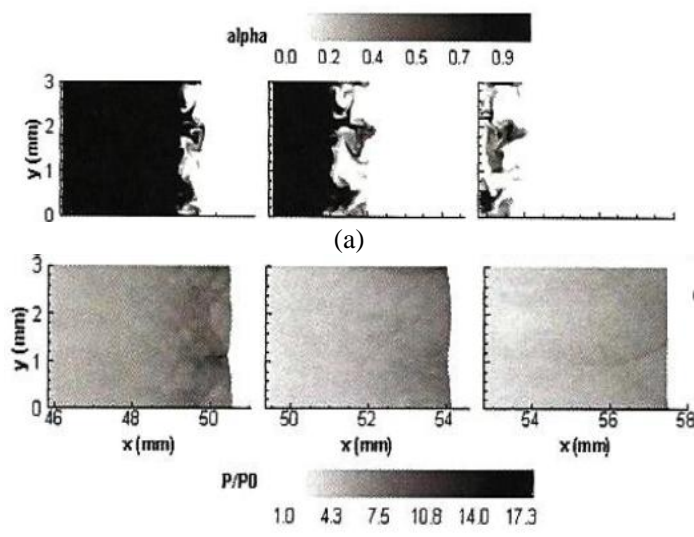

(b)

Fig. 5. $\alpha$ (a) and $P$ (b) for the standard setup. The time difference between the snapshot is $2.5(\mu \mathrm{s})$.

PIPO 0613192532384451576370

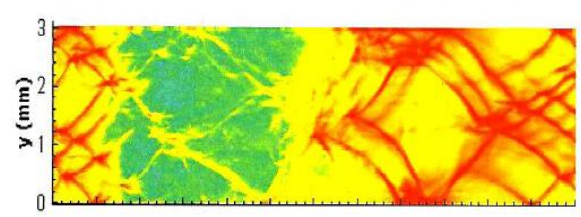

(a)

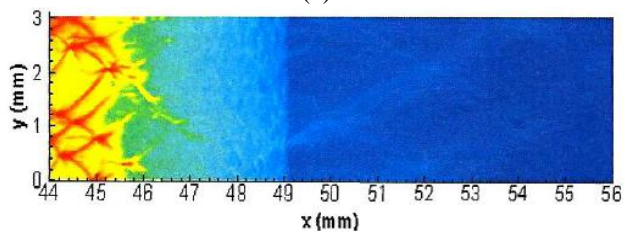

(b)

Fig. 6. Maximum pressure plots for cloud length $L=1 \mathrm{~mm}$ (a) and $L=4 \mathrm{~mm}$ (b). 


\section{B. Particle Cloud Size}

The influence of the size of the particle cloud was investigated by running simulations with cloud length $L=$ $1(\mathrm{~mm})$ and $L=4(\mathrm{~mm})$. The maximum pressure plot can be seen in Fig. 6. Two snapshot of pressure plots and particle distributions as a function of $x$ for $L=4(\mathrm{~mm})$ are found in Fig. 7 and 8 . The time difference between the snapshots is $2.5(\mu \mathrm{s})$. From these plots, it is clear that the volume fraction of particles increases somewhat behind the shock wave, caused by a compression of the particle cloud. This cloud indicates the formation of a $\beta$ layer, as discussed by Carvael et al. [7].
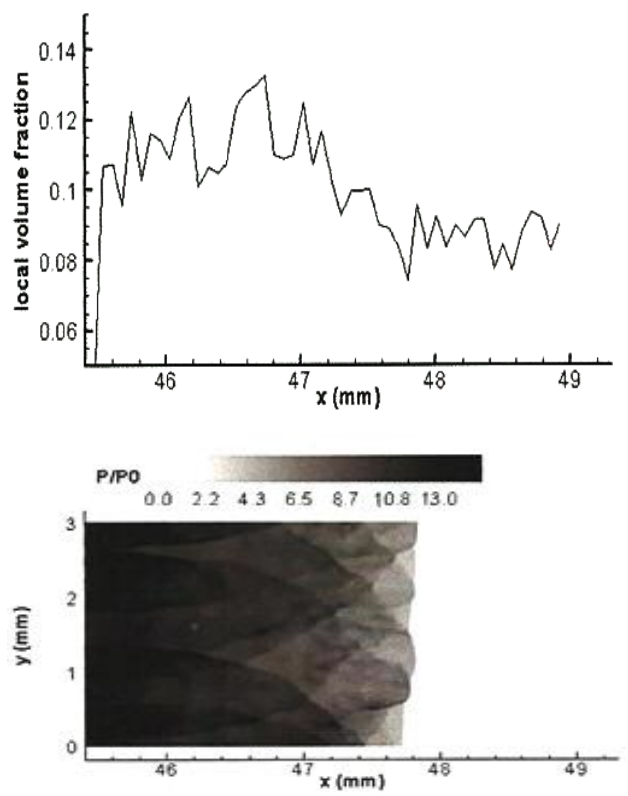

Fig. 7. Particle distribution and pressure plot for cloud length $L=4 \mathrm{~mm}$. The volume fraction is given in percent.
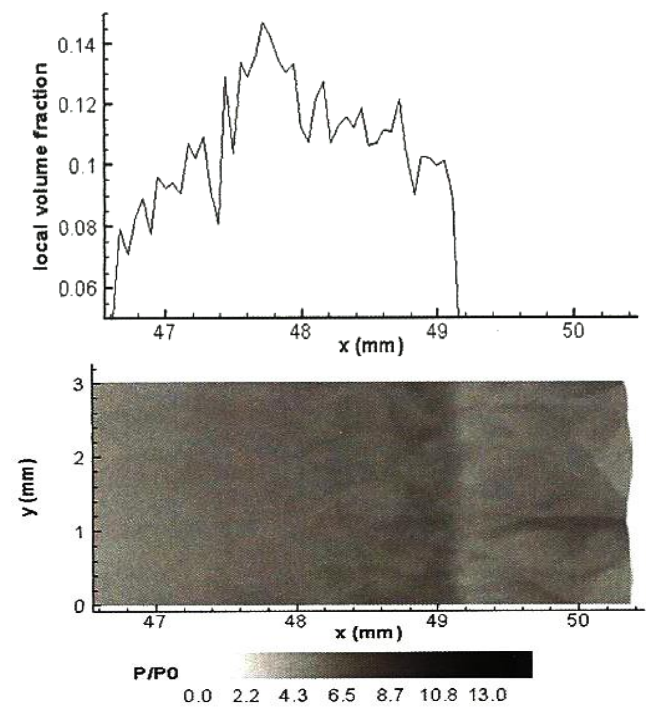

Fig. 8. Particle distribution and pressure plot for cloud length $L=4 \mathrm{~mm}$. The volume fraction is given in percent. This snapshot is taken $2.5 \mu$ s later than the one shown in Fig. 8.

\section{Volume Fraction}

The influence of the volume fraction of the particle could was investigated by running simulations with $\Phi=0.05 \%$ and $\Phi=0.2 \%$. The maximum pressure plot can be seen in Fig. 11 . A plot of the shock position and velocity as function of time is found in Fig. 10.

In the case of $\Phi=0.05 \%$, re-ignition occurs, and the detonation velocity is increased back to normal. These results are qualitatively in agreement with those found by Dong et al [16]. The similarity between the particle cloud length and volume fraction simulation is to be expected, because the same number of particles is used in both cases. However, the influence of inter-particle collisions could be of importance in the case of high particle volume fractions.
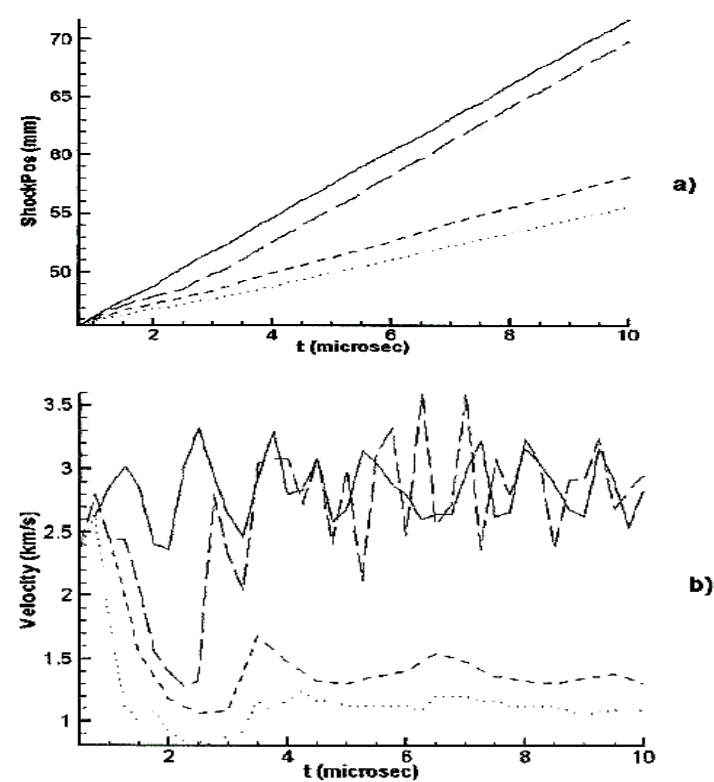

Fig. 9. Position of the shock front (a) and shock velocity (b) with no particles (solid line), $\Phi=0.1 \%$ (dashed line) and $\Phi=0.2 \%$ (dotted line).

PIPO 0613192532384451576370

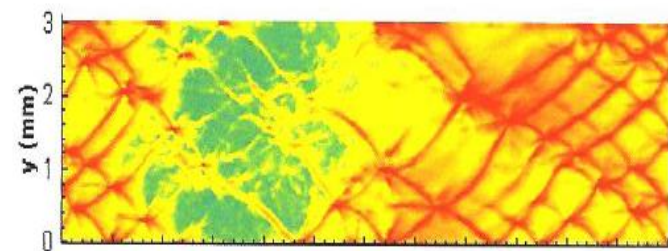

(a)

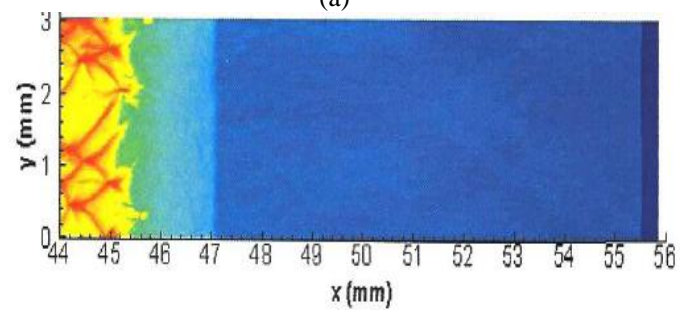

(b)

Fig. 10. Maximum pressure plot for volume fraction $\Phi=0.05 \%$ (a) and $\Phi=$ $0.2 \%$ (b)

\section{Particle Diameter}

The influence of the diameter of the particles was investigated by running simulations with $d_{p}=0.85(\mu \mathrm{m})$ and $d_{p}=0.85(\mu m)$ at constant volume fraction. The decreased particle diameter at constant volume fraction leads to increased total particle surface area, and faster heat exchange between the phases. The maximum pressure plot can be seen 
in Fig. 11. These plots show that the diameter of the particles have significant importance when it comes to detonation quenching. This is in agreement with Fedorov et al. [17], who concludes that particles with diameters in the range of $1 \mu \mathrm{m}$ ensures fairly effective quenching of a detonation.

PIPO
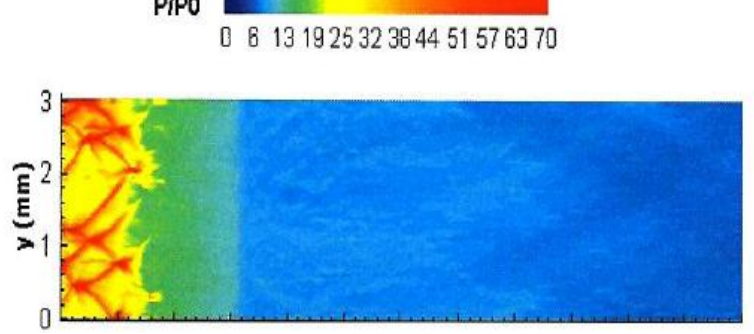

(a)

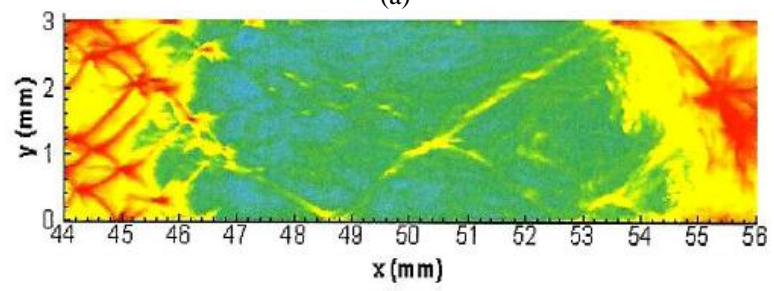

(b)

Fig. 11. Maximum pressure plots for particle diameters $d_{p}=0.85 \mu \mathrm{m}$ (a) and $d_{p}=0.85 \mu \mathrm{m}$ (b) at constant particle volume fraction.

\section{E. Particle Diameter}

An overview of the reduced pressure downstream of the particle cloud for the parameter variation calculations are presented in Table II. The lowest value of $p_{2} / p_{0}$ to give re-ignition was 12.4 , and the highest value with no re-ignition was 10.

TABLE II: $p_{2} / p_{0}$ FOR PARAMETER VARIATION SIMUlations. THE FirST Column Is the Standard Simulation. The value of THE Changed PARAMETER IS MADE DIMENSIONLESS BY DIVIDING ON THE STANDARD VALUE OF THE PARAMETER.

\begin{tabular}{|c|c|c|c|c|c|c|c|c|c|}
\hline $\begin{array}{c}\text { Parameter } \\
\text { changed }\end{array}$ & Std & $L$ & $L$ & $\Phi$ & $\Phi$ & $\rho_{v}$ & $\rho_{v}$ & $d_{v}$ & $d_{v}$ \\
\hline Value/std & - & 0.5 & 2 & 0.5 & 2 & 0.5 & 2 & 0.65 & 1.38 \\
\hline$p_{2} / p_{0}$ & 10 & 17.1 & 6 & 19 & 6.1 & 12.4 & 8.0 & 8.3 & 13.8 \\
\hline $\begin{array}{c}\text { Re } \\
\text { ignition }\end{array}$ & $\mathrm{N}$ & $\mathrm{N}$ & $\mathrm{Y}$ & $\mathrm{N}$ & $\mathrm{Y}$ & $\mathrm{Y}$ & $\mathrm{N}$ & $\mathrm{N}$ & $\mathrm{Y}$ \\
\hline
\end{tabular}

\section{F. Changes in Activation Energy}

PIPO $0 \quad 613192532384451576370$

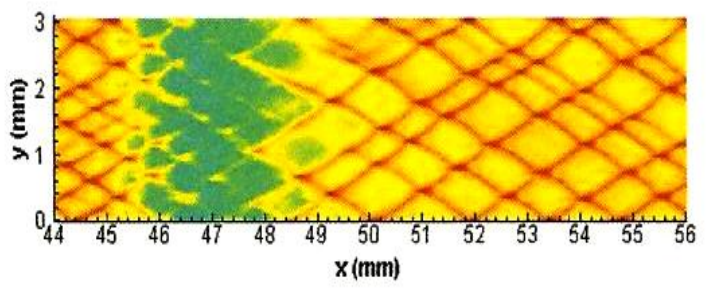

Fig. 12. Maximum pressure plot with particles. $E_{a}=104 \mathrm{~kg} / \mathrm{mol}$.

The maximum pressure plot with particles for
$E_{a}=29 \mathrm{~kg} / \mathrm{mol}$ is found in Fig. 13. If the heat loss caused by particle interaction is the main mitigating mechanism, it is reasonable to assume that decreasing activation energy reduces the effect of particles on the detonation structure, since the detonation propagation is less affected by temperature changes. Fig. 12 clearly supports this assumption. Due to stability issues, a solution for $E_{a}=104 \mathrm{~kg} / \mathrm{mol}$ with particles was not obtained, but it can be assumed that the mitigation effect of particle is substantially increased with this high activation energy.

\section{CONCLUding REMARKS}

A cloud of particles can have a substantial mitigating effect on a detonation wave, and cloud possibly causes total suppression. The effectiveness of the suppression is dependent on the parameters of the particle cloud, as well as the characteristics of the detonation wave.

The simulations indicate a critical value of the pressure downstream of the detonation cloud. With standard gas parameters, this critical pressure lies in the range of $p_{2} / p_{0}=10-12.5$. If more simulations were run, this critical pressure could be determined more precisely. The mitigation appears to be most effective with many small particles with large mass density. The heat loss caused by particle interaction can be assumed to be the main mitigating effect, but particle drag effects also play a significant role in the suppression of a detonation wave.

There is some weak cellular pattern in the pressure of the dampened shock wave downstream of the particle cloud. These are most likely some kind of residual shock from the cellular patterns in the original detonation. In simulation where re-ignition looks like it is initiated in the trajectory of these weak patterns.

For gases with low activation energy, the suppression appears to be less efficient.

The need for further investigations in detonation-particle interaction with Lagrangian particle model is definitely present. An inter-particle collision algorithm would make it possible to calculate dense particle clouds more accurately, and is a natural next step. This would also make it possible to further investigate the formation of a ( $\beta$-layer). When calculating the chemical reaction process in dense particle clouds, it is also necessary to account for porosity effects.

When investigating the suppression of a detonation, the need for a larger simulation area. A dynamic grid would be suitable to fully resolve the reaction process in the detonation front, and at the same time calculate the growing reaction zone behind the initial shock where lower resolution is required. It could be necessary to apply some kind of turbulence modeling in this region, as the characteristic velocities are lower than in the in the initial detonation wave. A larger simulation area would make it possible to investigate the re-ignition of a detonation more accurately. The role of weak cellular patterns in the re-ignition of a detonation wave should also be studied in more detail.

There is an urgent need for developing a better model for particle drag and heating, since these are the two governing mechanisms in detonation-particle interaction. A better 
understanding of the dynamic viscosity and heat conductivity of reactive gases at high temperatures and pressures is needed to develop such a model. Experiments with irregular particle shapes should also be conducted, as Kovtun et al. [18] showed structural changes in particles caused by shock waves.

Lagrangian approach to particle modeling is easy adaptable to simulating detonation suppression by means of adding suspended liquid droplets. This could prove to be a very effective method of detonation quenching, since additional heat is needed to evaporate the droplets.

As pointed out by Fedorov et al. [17], experimental reports of detonation suppression by means of inert particles are rather scattered. In the experiments carried out by Dong et al. [16], some quantitative difference occurred between experiments and calculations. There is an urgent need for experimental investigation, in order to validate simulation of detonation suppression. However, the practical limitations of such experiments are pointed out by Dong et al. They argue that the complete dispersion of a cloud of particle is very difficult, as some agglomeration and wall effects cannot be avoided. The present studies have shown that the effectiveness of detonation suppression is very sensitive to particle size. This implies that an effective suppression system must ensure very good dispersion of the particles. As different particle materials have different physical structures and properties, particle material variation experiments should carried out to find suitable suppression agents.

\section{ACKNOWLEDGMENT}

This paper is a part of master thesis research in field of Aerospace Engineering (propulsion) in Ferdowsi University of Mashhad, Iran.

\section{REFERENCES}

[1] R. K. Eckhoff, Explosion Hazards in the Process Industries, Gulf Publishing Company, 2005.

[2] V. P. K. S. K. V. M. Bioko, A. Papyrin, S. Poplavsky, and V. Fomin, "Shock wave interaction with a cloud of particles," Shock Wave, vol. 7, pp. 275-285, 1997.

[3] Y. X. B. Wang and L. Qi, "Shock-induced near -wall two-phase flow structure over a micron-sized particles bed," Shock Wave, vol. 15, pp. 363-373, 2006

[4] A. H. M. Parmar and S. Balachandar, "Modeling of the unsteady force for shockparticle interaction," Shock Wave, vol. 19, pp. 317-329, 2009.

[5] J. W. W. J. E. S. Oran, E. I. Stefaniw, M. H. Lefebvre et al., "A numerical study of a two dimensional h2-o2 detonatio using a detailed chemical reaction model," Combustion and Flame, vol. 113, no. 1-2, pp. 147-163, 1998 .

[6] D. Desbordes, E. S. Oran, and V. N. Gamezo, "Two-Dimensionalreactive flow dynamics in cellular detonation waves," Shock waves, vol. 9, pp. 11-17, 1999.
[7] G. T. R. Carvel and C. Brown, "Some observations of detonation propagation through a gas containing dust particles in suspension," Shock wave 13, pp. 83-89, 2003.

[8] P. E. DesJardin, M. R. Baer, K. P. Ruggirello, and E. S. Hertel, "Modeling of particle compressibility and ignition from shock focusing," Combustion Theory and Modeling, vol. 14, pp. 41-67, 2010.

[9] K. K. S. Cheatham, "Numerical modeling of liquid-fuelled detonations in tubes," Combustion Theory and Modeling, vol. 9, no. 1, pp. 23-48, 2005 .

[10] S. M. Frolov, "Detonation initiation and DDT: Experiments and numerical simulations," in Proc. the 5th International Seminar on Fire and Explosion Hazards, 2007.

[11] M. S. C. Crowe and Y. Tsuji, Multiphase Flow with Droplet and Particles, CRC Press, 1998.

[12] J. R. C. P. A. Fomin, "Effect of chemically inert particles on thermodynamic characteristics and detonation of a combustible gas," Combustion Science and Technology, vol. 181, no. 8, pp. 1038-1064, 2009.

[13] S. U. D. Balapanov, "Role of interphase interactions during gas detonation in inert porous medium," Technical Physics Letters, vol. 36, pp. 618-622, 2010.

[14] M. V. Papalexanderis, "Influence of inert particles on the propagation of multidimensional detonation waves," Combustion and Flame, vol. 141, no. 3, pp. 216-228, 2005.

[15] T. G. C. S. Chapman, The Mathematical Theory of Non-Uniform Gases, Cambridge University Press, 1970.

[16] B. F. G. Dong, B. Xie, and J. Ye, "Experimental investigation and numerical validation of explosion suppression by inert particles in large-scale dust," The Combustion Institute, vol. 30, no. 2, pp. 2361-2368, 2005.

[17] D. T. A. Fedorov and I. Bedarev, "Mathematical modeling of detonation suppression in hydrogen-oxygen mixture by inert particles," Combustion, Explosion, and Shock Waves, vol. 46, pp. 332-343, 2010

[18] M. V. K. V. I. Kovtun, V. V. Garbuz, S. M. Zakharov, N. V. Zaitseva, O. A. Shmatko, M. V. Bober, and A. Demidik, "Structural and phase changes in cooper, cobalt, and titanum oxidpowders subjected to shock waves," Powder Metallorgy and Metal Ceramics, vol. 47, pp. 622-628, 2008 .

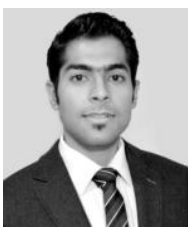

Hesam Shafiee is a M.Sc. student of aerospace engineering (propulsions) in Ferdowsi University of Mashhad. Hesam Shafiee was born at Tehran on Sept. 6, 1987, and received B.Sc. in fluid mechanic from Ferdowsi University of Mashhad in 2010. His major fields of study are multiphase flow and computationa fluid dynamics. He has two papers in national scientific conferences. Now he is doing research in subject of small droplets behavior in co-operation with Statoil and Acona Flow in Telemark University College. $\mathrm{He}$ is also working as an analyst in Stressman Engineering AS. in Herøya Industry Park , Porsgrunn, Norway.

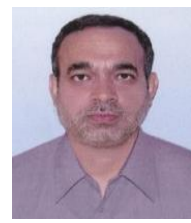

Mohamad Hassan Djavareshkian was born at Mashhad on Aug. 24, 1962, and received B.Sc. in fluid mechanic from Iran University of Science \& Technology (Iran) in 1982, M.Sc. in fluid mechanic from Liverpool University (England) on 1993 and $\mathrm{PhD}$, in computational fluid dynamics and aerodynamics from Imperial College of Science and Technology (England) in 1997. His major fields of study are computational fluid dynamics and aerodynamics. His publication is more than one hundred papers in international journal and presented at National and International Scientific conferences. A few PhD theses and more than forty M.Sc. theses have done under his supervision. Now he's working at the Mechanical Engineering Department of Ferdowsi University of Mashhad. 